\title{
Analysis of dentin bond strength and bonding interface using alternative conditioners
}

\section{Beatriz O. Sahadi*, Maicon Sebold, Carolina B. André, Marcelo Giannini.}

\section{Abstract}

This study evaluated the effects of alternative dentin conditioners on the bond strength, failure mode, and morphology of the bonding interface. Forty teeth were used for the bond strength test $(n=8)$. The Adper Single Bond 2 (3M Oral Care) adhesive was applied on moist (control), and air-dried (experimental) etched dentin after treatment with $37 \%$ phosphoric acid (PA), $3 \%$ aluminum nitrate $+2 \%$ oxalic acid (NA), $6.8 \%$ ferric oxalate $+10 \%$ citric acid (FO), or $10 \%$ citric acid (CA). Data normality was analyzed by one-way ANOVA, followed by Tukey's test $(\alpha=0.05)$. Failure modes were observed by scanning electron microscopy (SEM). The morphology of the bonding interface $(n=2)$ was analyzed by confocal laser scanning microscopy (CLSM). Wet-bonding with PA led to higher bond strength than the other groups. Bond strengths of NA, FO, and CA did not differ among themselves $(p>0.05)$. Mixed failures were predominant in the control and CA groups, and adhesive failures were predominant for NA and FO. In the control group, a hybrid layer with long resin tags could be obtained. For NA and CA, the formation of poor-quality hybrid layers was evident, while no hybridization occurred in the FO group. Adhesive application on air-dried dentin etched with the tested conditioners led to poor hybrid layer formation and lower bond strengths compared to the wet-bonding with PA.

\section{Key words:}

Dentin Adhesives, Confocal Miscroscopy, Tensile Strength.

\section{Introduction}

Dentin bonding by acid-etching requires the substrate to be kept moist prior to adhesive application. Water increases the complexity of the adhesive procedure, impairs the polymerization of the bonding agent, and leads to hydrolysis of the adhesive. Also, phosphoric acid can activate dentin metalloproteinases (MMPs) and cause changes in the collagen molecular arrangement that favor the action of these enzymes. Considering all these problems related to the wet-bonding approach with phosphoric acid, conditioning agents that allow drybonding to dentin, while inhibiting MMP activity should be studied.

\section{Results and Discussion}

Alternative dentin conditioners (3\% aluminum nitrate + $2 \%$ oxalic acid; $6.8 \%$ ferric oxalate $+10 \%$ citric acid; and $10 \%$ citric acid) were tested with an etch-and-rinse adhesive (Adper Single Bond 2, 3M Oral Care) applied on dry, etched dentin, and compared to a control group, in which the adhesive was applied on moist dentin after $37 \%$ phosphoric acid-etching. The following methodologies were used: (1) dentin microtensile bond strength test; (2) failure mode analysis by SEM; and (3) bonding interface morphology analysis by MCVL.

Table 1. Mean ( $\pm S D$ ) dentin microtensile bond strength according to dentin treatment.

\begin{tabular}{lc}
\hline \multicolumn{1}{c}{ Dentin Treatment } & $\begin{array}{c}\text { Bond Strength } \\
\text { (in MPa) }\end{array}$ \\
\hline Control (PA wet-bonding) & $50.8( \pm 6.5) \mathrm{A}$ \\
$3 \%$ Aluminum nitrate $+2 \%$ oxalic acid & $26.8( \pm 9.1) \mathrm{B}$ \\
$6.8 \%$ Ferric oxalate $+10 \%$ citric acid & $32.4( \pm 12.8) \mathrm{B}$ \\
$10 \%$ Citric acid & $35.4( \pm 9.1) \mathrm{B}$ \\
\hline
\end{tabular}

Means followed by distinct letters differ from each other ( $p$ $\leq 0.05$ ).

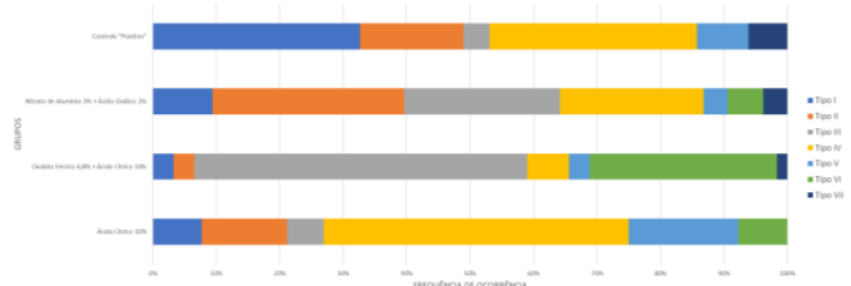

Figure 1. Failures modes (in \%) according to dentin treatment.

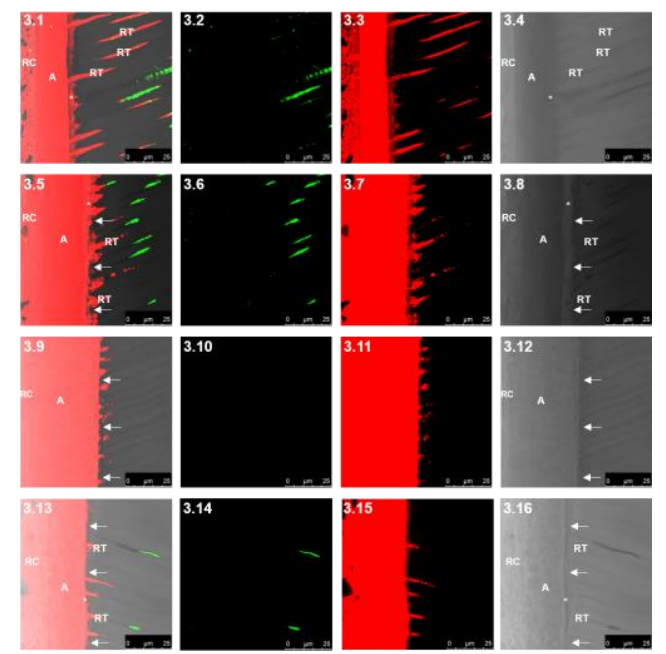

Figure 2. MCVL micrographs of the Bonding interface. The first column represents the overlap of the second (dentin fluorescence), the third (fluorescence of the dye in the adhesive resin), and the fourth (transmission mode) columns. (3.1 - 3.4) control; (3.5 - 3.8) 3\% aluminum nitrate $+2 \%$ oxalic acid; (3.9 - 3.12) $6.8 \%$ ferric oxalate $+10 \%$ citric acid; $(3.13-3.16) 10 \%$ citric acid. $\left(^{*}\right)$ indicates hybridization zones; arrows $(\rightarrow)$ indicate etched zones that were not infiltrated by the adhesive $\mathrm{RC}$ - resin composite; A - adhesive layer; RT - resin tags.

\section{Conclusions}

None of the tested conditioners could create dentin bonds comparable to the phosphoric acid. However, both solutions containing citric acid still presented bond strengths over $30 \mathrm{MPa}$, with hybrid layer formation in the $10 \%$ citric acid group. Future investigations on the use of citric acid as a dentin conditioner would be interesting.

Acknowledgement

\section{PIBIC-CNPq}

